\title{
EFECTO DEL CAMBIO EN LA CONDICIÓN CORPORAL, RAZA Y NÚMERO DE PARTOS EN EL DESEMPEÑO REPRODUCTIVO DE VACAS LECHERAS ${ }^{1}$
}

\author{
Elmer Edgardo Corea-Guillén ${ }^{2}$, Juan Francisco Alvarado-Panameño ${ }^{2}$, Ludwing Vladimir Leyton-Barrientos ${ }^{2}$
}

\begin{abstract}
RESUMEN
Efecto del cambio en la condición corporal, raza y número de partos en el desempeño reproductivo de vacas lecheras. Este estudio fue conducido en el El Salvador con el objetivo de conocer el comportamiento de la condición corporal, la urea y colesterol sanguíneo y determinar la relación entre la pérdida de condición corporal posparto, el grupo racial y los partos en las variables reproductivas de vacas lecheras. Se utilizaron 40 vacas Holstein y 40 vacas cruzadas (Holstein/Brown swiss/Cebú) en su periodo preparto. Se midió la condición corporal y se muestreó la leche y la sangre cada dos semanas desde 45 días preparto hasta 56 días posparto y se obtuvo además la información de número de partos y eventos reproductivos. Se determinó progesterona en leche por radioinmunoensayo de fase sólida y en los sueros sanguíneos se midió nitrógeno ureico y colesterol por espectofotometría. La pérdida de condición corporal posparto fue mayor en vacas Holstein $(0,93 \pm 0,49)$ que en vacas cruzadas $(0,65 \pm 0,51)$. La duración del anestro fue mayor en vacas Holstein (63,8 \pm siete días) que en vacas cruzadas $(37,24 \pm$ seis días $) \mathrm{p}<0,05$, y en vacas con mayor pérdida de condición corporal $>1(63,67 \pm 36$ días $)$ que en vacas con menor pérdida $0,5-1$ (46,4 $\pm 24,42$ días), < 0,5 $(36,7 \pm 15,7$ días $) \mathrm{p}<0,05$. No se encontró efecto de la pérdida de condición, grupo racial ni del número de partos en los servicios por concepción ni en los días abiertos. La genética Holstein y la pérdida de condición corporal se relacionaron negativamente con la duración del anestro.
\end{abstract}

Palabras clave: Urea, evaluación reproductiva, anestro, fertilidad, balance energético.

\begin{abstract}
Effect of change in body condition, breed and parity on the performance of dairy cows. This study was carried in the central area of El Salvador with the aim of knowing the behavior of the body condition, the blood urea and cholesterol and to determine the relationship among the postpartum body condition loss, genetics and parity in reproductive parameters of dairy cows. For this 40 Holstein and 40 crossbred (Holstein/Brown swiss/zebu) prepartum cows were used. Body Condition Score was measured and milk and blood samples was taken every two weeks since 45 days prepartum to 56 days postpartum, information was obtained from the number of births and reproductive events. Milk progesterone were determined using solid phase radioimmunoassay and the serum urea nitrogen and cholesterol was measured by espectofotometry. The pospartum body condition loss was higher in Holstein cows $(0.93 \pm 0.49)$ than in crossbred cows $(0.65 \pm 0.51)$. The length of anestrus was higher in Holstein cows (63.8 \pm seven days) than in crossbred cows (37.24 \pm six days) $\mathrm{p}<0.05$, and in cows with greater loss of body condition $>1(63.67 \pm 36$ days ) than in cows less lost 0.5-1 (46.4 \pm 24.42 days), $<0.5$ $(36.7 \pm 15.7$ days) $\mathrm{p}<0.05$. The effect of BCS, genetics or parity in the services per conception or in the open days was not significant. It is concluded that the genetic Holstein and loss of body condition relate negatively with the length of anestrus.
\end{abstract}

Key words: Urea, reproductive performance, anoestrous, fertility, energy balance.

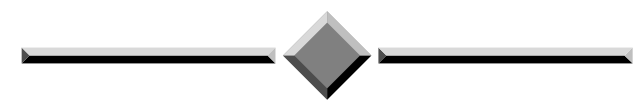

Recibido: 11 de marzo, 2008. Aceptado: 25 de agosto, 2008.

2 Departamento de Zootecnia, Facultad Ciencias Agronómicas, Universidad de El Salvador. elmercorea@hotmail.com; ludleyton@yahoo.com; jfranciscosv@yahoo.com 


\section{INTRODUCCIÓN}

Estudios recientes en bovinos mostraron que la eficiencia reproductiva es baja en El Salvador en vacas productoras de leche. Se encontraron dos diferentes situaciones, una en fincas con vacas con alto encaste Holstein donde se reinicia la actividad ovárica muy pronto después del parto pero la concepción se retrasa $y$ otra en fincas de bajo encaste europeo, en que los periodos de anestro prolongados parecen ser el problema más relevante. Se observó además que en la distribución de las variables reproductivas dentro de una misma finca, existe un grupo importante de vacas con mal desempeño cuyas determinantes no fueron estudiadas (Corea et al. 2004).

Existen varios factores que afectan la reproducción, el medio ambiente es uno de los más importantes (Gwazdauskas et al. 1974). El calor y la elevada humedad, afectan negativamente la reproducción en vacas lecheras (Flamembaum 1997). Vacas con mayores producciones tienden a tener menor desempeño reproductivo, principalmente retraso en la actividad ovárica posparto y bajas en la tasa de concepción (Laben et al. 1982, Nebel y Mcgilliard 1993, Butler 1998) Sin embargo, no se ha encontrado una asociación entre la correlación de la heredabilidad para producción y fertilidad (Raheja et al. 1989). De manera que el conocido menor desempeño reproductivo de las altas productoras es más influenciado por factores de manejo nutricional y reproductivo.

La energía es el nutriente que más frecuentemente limita la reproducción. Se ha descrito una relación inversa entre el balance energético (BE) negativo, el reinicio de la actividad ovárica y la fertilidad (Randel 1990, Butler y Smith 1989), y ya que el BE puede ser evaluado por medio de calificación de condición corporal (Edmonson et al. 1989) ha sido posible establecer ensayos que demuestran que vacas con mayores pérdidas de condición, tienen menor desempeño reproductivo (Domec et al. 1997b, Delgado et al. 2004).

El aporte proteico puede también tener un rol importante. Se ha reportado una relación antagónica entre el consumo de proteína y el desempeño reproductivo (Ferguson y Chalupa 1989, Hojman et al. 2004), así como la relación entre medición de urea en sangre y leche como indicadores del consumo y metabolismo proteico (Baker et al. 1995, Hof et al. 1997).

Las prácticas de manejo inadecuadas predisponen a infecciones, retenciones de placenta, servicios por concepción y días abiertos (Coleman et al. 1985) lo cual puede ser también relevante en algunos casos de deficiente desempeño reproductivo.

El objetivo del estudio fue determinar el cambio en condicion corporal y los niveles de metabolitos sanguíneos en el posparto y establecer el efecto del cambio en condición corporal, el grupo racial y número de pariciones con los parámetros reproductivos de vacas lecheras en dos fincas de la región paracentral de El Salvador.

\section{MATERIALES Y MÉTODOS}

El estudio se llevó a cabo en dos lecherías de la región paracentral de El Salvador, en el municipio de Olocuilta y el cantón de Comalapa en el departamento de La Paz. Se seleccionaron 80 vacas y novillas con historias reproductivas normales (Cuadro 1), tuvo una duración de 12 meses, a partir de julio de 2003. Se inició un mes y medio antes del parto y se terminó con el segundo servicio/celo de cada vaca.

Cuadro 1. Características de las vacas estudiadas. La Paz, El Salvador. 2004.

\begin{tabular}{lcccccc}
\hline \multicolumn{1}{c}{ Encaste } & $\begin{array}{c}\text { Vacas en } \\
\text { ordeño }\end{array}$ & $\begin{array}{c}\text { Novillas } \\
\text { incluidas }\end{array}$ & $\begin{array}{c}\text { Vacas } \\
\text { incluidas }\end{array}$ & Manejo & $\begin{array}{c}\text { Producción } \\
\text { kg/día }\end{array}$ & $\begin{array}{c}\text { Peso aproxi- } \\
\text { mado (kg) }\end{array}$ \\
\hline Holstein/ & & 15 & 25 & Estabulado & 15 & 550 \\
B. Swiss/Cebú & 80 & 13 & 27 & Estabulado & 20 & 450 \\
Holstein & 130 & 15 & & & \\
\hline
\end{tabular}


Las vacas Holstein pertenecían a un hato purificado en base al uso continuo de semen importado durante muchos años, alcanzando el tipo característico de la raza. Las vacas en el hato cruzado, provenían de una base Cebú que fue mejorado con semen Holstein y Brown Swiss durante aproximadamente 10 años; encontrándose variación de tipos.

\section{Muestreo}

Leche: A partir de 15 días postparto hasta el segundo celo/inseminación o diagnóstico de preñez, se tomó una muestra semanal de leche en un recipiente de $10 \mathrm{ml}$ con preservante de formaldehido. Estas fueron transportadas y almacenadas a $4{ }^{\circ} \mathrm{C}$; se separó la grasa en centrífuga a 3.000 RPM durante 10 minutos y la fracción libre de grasa se almacenó en crioviales $\mathrm{a}-10{ }^{\circ} \mathrm{C}$.

Colección de datos: Se obtuvo información de las vacas referente al número de partos y los eventos reproductivos: fecha de parto, fechas de celo, fechas de servicio y de concepción con el fin de calcular parámetros reproductivos; se tomó calificación de condición corporal (CCC) cada dos semanas: desde 42 días preparto hasta 56 días posparto (según escala de cinco puntos propuesta por Edmonson et al. 1989).

Sangre: Las vacas fueron sangradas cada dos semanas iniciando 42 días preparto hasta 56 días posparto. Las muestras fueron tomadas por punción de la vena yugular en tubos estériles de $10 \mathrm{ml}$ con vacío y sin anticoagulante, fueron centrifugadas a 3.000 RPM durante 10 minutos. Los sueros se depositaron en crioviales por duplicado y se guardaron $\mathrm{a}-10^{\circ} \mathrm{C}$.

\section{Determinaciones de Laboratorio}

La progesterona fue analizada en las muestras de leche por Radioinmunoensayo de fase sólida (Self Coating, OIEA). El límite de detección fue 0,1 nmol/1.

En los sueros obtenidos se analizaron Nitrógeno Ureico Sanguíneo (NUS) colesterol (LABTEST DIAGNOSTICA) por espectrofotometría de luz visible.

\section{Variables evaluadas}

Los valores de condición corporal, NUS y colesterol fueron graficados para el período pre y postparto.

Las variables reproductivas entre el parto y el reinicio de la actividad ovárica o anestro (considerando inicio de la actividad ovárica la primera elevación de progesterona arriba de 2,9 nmol/l. Bolaños y Molina (1992), intervalo parto-concepción (días abiertos) y número de inseminaciones por concepción fueron calculados.

\section{Análisis estadístico}

Los datos fueron analizados utilizando el Modelo General Lineal (GLM) del programa estadístico $\mathrm{SAS}^{\circledast}$, el modelo incluyó los efectos del grupo racial, el cambio en condición corporal después del parto y las pariciones en la duración del anestro, servicios por concepción y días abiertos. Se realizó a cada variable la prueba de diferencia mínima significativa de medias.

En algunas de las vacas elegidas para el estudio no se obtuvo la información esperada para las variables días abiertos y servicios por concepción ya sea por muerte (cinco Holstein), venta (cinco cruzadas y dos Holstein) o falta de preñez (cuatro cruzadas y cinco Holstein).

\section{RESULTADOS Y DISCUSIÓN}

\section{Condición corporal}

Se ha reportado que las vacas que paren con mayor CCC tienen las mayores pérdidas de condición posparto (Ruegg et al. 1992). Sin embargo, en este estudio la condición corporal preparto fue menor y la pérdida en condición corporal desde el parto a los 56 días posparto fue mayor en vacas Holstein $(0,93 \pm$ $0,49)$ que en cruzadas $(0,65 \pm 0,51)$ Figura 1 . Pryce et al. 2001 también encontraron que las vacas con mayor genética Holstein tuvieron mayores pérdidas de condición y que la mayor pérdida de condición se relacionó 


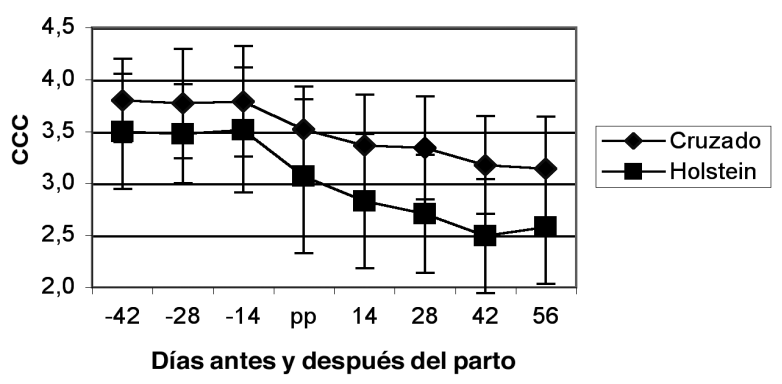

Figura 1. Cambio en condición corporal de ganado Holstein y cruzado (promedio \pm desviación estándar) desde 42 días preparto hasta 56 días postparto. $\mathrm{p}=$ parto (4,5 \pm 1,96 días postparto). La Paz, El Salvador. 2004.

negativamente con el desempeño reproductivo. La mayor pérdida de condición corporal ha sido relacionada con mayores producciones que demandan más energía y movimiento de las reservas corporales (Domec et al. 1997a) ya que existe una correlación directa $(r=-0,8)$ entre producción láctea y balance energético (Butler y Smith 1989). Las vacas que tienen mayor pérdida de condición, presentan además más incidencia de mastitis y enfermedades metabólicas como desplazamiento de abomaso, fiebre de leche y cetosis (Kim y Suh 2003), aunque no se estudió la incidencia de enfermedades en relación con la pérdida de condición, la muerte de vacas en el período de estudio en el grupo con mayor pérdida de condición podría relacionarse con la ocurrencia de estas enfermedades.

\section{Nitrógeno Ureico en Sangre}

El nitrógeno ureico en sangre (NUS) y en leche (NUL) ha sido usado como herramienta para evaluar la nutrición proteica en vacas y su relación con la fertilidad (Ferguson et al. 1993, Baker 1995, Hof et al. 1997, Godden et al. 2001). Las vacas que consumen más proteína, poseen niveles sanguíneos y uterinos más altos de urea (Jordan et al. 1983). Los niveles de NUS encontrados fueron mayores en las vacas Holstein, quienes tienen mayor producción y reciben más proteína en la dieta que las cruzadas (18 vs 15\%), y

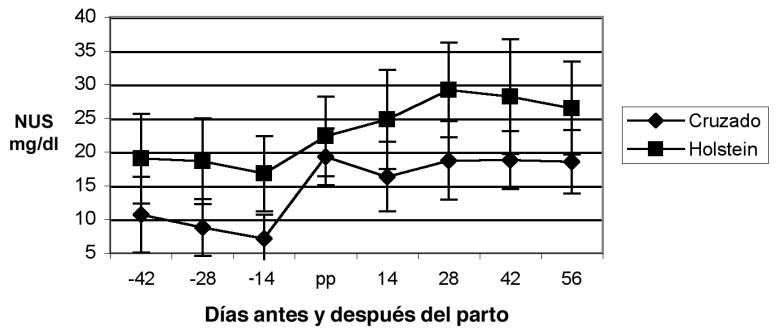

Figura 2. Concentraciones de Nitrógeno Ureico Sanguíneo NUS (promedio \pm desviación estándar) desde 42 días preparto hasta 56 días posparto. $\mathrm{p}=$ parto $(4,5$ $\pm 1,96$ días posparto) en ganado Holstein y cruzado. La Paz, El Salvador. 2004.

fueron mayores en ambos grupos después del parto cuando reciben mayor suplementación (Figura 2).

En un estudio reciente, también se encontró niveles de NUS por encima de los valores considerados normales $(12-18 \mathrm{mg} / \mathrm{dl})$ y excesos de proteína en las raciones de ocho ganaderías lecheras similares en las estaciones seca y lluviosa (Zavala et al. 2005).

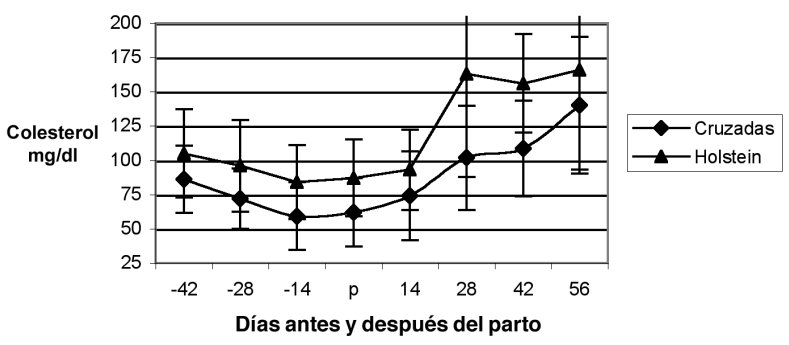

Figura 3. Concentraciones de colesterol de ganado Holstein y cruzado (promedio \pm desviación estándar) desde 42 días preparto hasta 56 días posparto. $\mathrm{p}=$ parto (4,5 \pm 1,96 días postparto). La Paz, El Salvador. 2004.

\section{Colesterol}

Se ha reportado que el colesterol sanguíneo aumenta en el posparto de las vacas lecheras en proporción a la producción láctea (Ruegg et al. 1992). Coincidentemente los valores de colesterol sanguíneo encontrados fueron mayores en las vacas con mayor producción y se incrementaron después del parto (Figura 3). 


\section{Anestro}

La duración del anestro se vio afectada por el efecto del encaste $(\mathrm{p}<0,0001)$ y de la condición corporal $(\mathrm{p}<0,05)$ pero no por las pariciones $(\mathrm{p}=0,12)$ de las vacas estudiadas.

Las diferencias encontradas entre vacas Holstein y cruzadas en el reinicio de la actividad ovárica posparto (Cuadro 2), pueden ser resultado de la adaptación de los grupos raciales a las condiciones ambientales o a

Cuadro 2. Efecto de grupo racial, el cambio en condición corporal y pariciones en los servicios por concepción. La Paz, El Salvador. 2004.

\begin{tabular}{lccc}
\hline \multicolumn{1}{c}{ Factor } & N & $\begin{array}{c}\text { Servicios por } \\
\text { concepción (de } \\
\text { vacas preñadas) }\end{array}$ & $\begin{array}{c}\text { Desviación } \\
\text { estándar }\end{array}$ \\
\hline Grupo racial & 30 & $2,13 \mathrm{a}$ & 1,07 \\
Cruzadas & 29 & $1,51 \mathrm{~b}$ & 0,68 \\
Holstein & & & \\
Cambio en Con- & & $2,06 \mathrm{a}$ & 1,16 \\
dición Corporal & & $1,81 \mathrm{a}$ & 1,00 \\
$0,0-0,49$ & 15 & $1,68 \mathrm{a}$ & 0,71 \\
$0,5-0,99$ & 22 & & 1,04 \\
$>1$ & 22 & $1,83 \mathrm{a}$ & 0,89 \\
Pariciones & & $1,82 \mathrm{a}$ & \\
Primeriza & 24 & 35 & \\
Adulta & & & \\
\hline
\end{tabular}

Medias con la misma letra en la misma columna no difieren significativamente, según DMS, $\mathrm{p}<0,05$.

las diferencias en criterios de manejo, principalmente el manejo reproductivo y nutricional (Zarco 2000). A menudo se asocia la mayor producción de leche durante el posparto con un retorno a la ciclicidad más lento (Staples et al. 1990). Vacas con mayores producciones tienen su primer estro después que aquellas de más baja producción (Whitmore et al. 1974).

El rol del cambio en condición corporal en el reinicio de la actividad ovárica (Cuadro 2), estaría reflejando el efecto del balance energético negativo. Las vacas de alta producción demandan mayor cantidad de nutrientes lo cual las coloca fácilmente en balance energético negativo (Bach 2002). Para que una vaca ovule temprano en el posparto se debe restablecer la actividad folicular en el ovario y esto depende de la restauración de la secreción pulsátil de hormona luteinizante (LH) y el balance energético (Latrille 1993). Las vacas lactantes independientemente de su nivel de producción se encuentran en balance energético negativo durante las primeras seis a ocho semanas retrasando el comienzo de actividad ovárica y disminuyendo eventualmente la eficiencia reproductiva (Butler y Smith 1989).

También problemas como cojeras, mastitis u otras enfermedades metabólicas estarían relacionadas directamente con la condición corporal. Se observó que las vacas con problemas de salud normalmente tenían condiciones corporales bajas o tuvieron mayor pérdida. La incidencia de estas enfermedades es mayor en los primeros 50 días postparto y puede afectar la eficiencia reproductiva, el peso, la condición corporal y la sobrevivencia del animal en el rodeo (De La Sota et al. 2001).

Aunque estudios previos han reportado que las vacas primerizas tienen un balance energético menor y mayores intervalos a la primera ovulación (Lucy et al. 1992, Cavestany 2000a), en este estudio no se encontró diferencia entre vacas primerizas y adultas en el retorno a la actividad ovarica (Cuadro 1). Probablemente, los niveles de producción de las vacas a través de este estudio (15 kg las cruzadas, $20 \mathrm{~kg}$ las Holstein), no causaron un balance energético tan negativo como el de otros estudios donde sí hubo diferencias.

\section{Servicios por concepción}

Se encontró efecto del grupo racial $(\mathrm{p}<0,05)$ en los servicios por concepción, pero el cambio en condición corporal $(\mathrm{p}=0,70)$ y las pariciones $(\mathrm{p}=0,92)$ no tuvieron efectos importantes sobre éstos en las condiciones de este estudio.

Estudios previos (Ropstad y Refsdal 1987, Randel 1990, Hojman et al. 2004, Melendez et al. 2000) han 
indicado que vacas que reciben dietas más altas en proteína tienen menor fertilidad. La fertilidad de las vacas decrece por arriba de $20 \mathrm{mg} / \mathrm{dl}$ de urea serica (Ferguson et al. 1993, Butler 1998). Se ha señalado un efecto negativo en la sobrevivencia de gametos y embriones por la concentración de urea (Jordan et al. 1983) y alteración del pH (Butler 1998) en el fluído uterino y niveles plasmáticos bajos de progesterona antes del celo (Carrol et al. 1988) como las posibles causas de esta baja fertilidad. Además, se ha reportado que las vacas con mayores producciones tienen menor fertilidad (Butler 1998, Bach 2002). La menor cantidad de servicios en las vacas con mayor aporte proteico, mayor producción y mayores niveles de urea sanguinea parece contradecir estos reportes. Sin embargo, se ha relacionado la fertilidad con los criterios usados para elegir una vaca a inseminar así como la habilidad del inseminador con la fertilidad (Badinga et al. 1985, Coleman et al. 1985), el hecho de que en la finca con menos servicios por concepción se ofrezca un incentivo económico al inseminador por su efectividad, podría explicar en parte estos resultados. Por otra parte, la tasa de concepción decrece en vacas cuando la temperatura en el día de la inseminación excede los $30^{\circ} \mathrm{C}$ (Badinga et al. 1985) y los meses mas cálidos se asocian negativamente con las tasas de concepción (Gwazdauskas et al. 1974), ésto también puede ser una ventaja para las vacas Holstein que estaban en un lugar donde las temperaturas son un poco menores que donde estaban las vacas cruzadas (23 vs $26^{\circ} \mathrm{C}$ ). Además, la concentración energética de la dieta ha sido identificada como modificador de la proteína en la reproducción (Ferguson y Chalupa 1989), y no se estudió la relación energía-proteína.

Se ha reportado una relación inversa entre la pérdida de condición corporal y la producción con la probabilidad de concepción (Ruegg et al. 1992, Domec et al. 1997b). Sin embargo, en este estudio, aunque no se encontró un efecto entre la pérdida de condición corporal y los servicios por concepción, parece haber una tendencia inversa, es decir, que a mayor pérdida de condición, menos servicios (Cuadro 3), esto podría relacionarse con que las vacas con mayor pérdida de condición tienden a tener anestros más prolongados y al reiniciar la actividad ovárica tardíamente, suelen tener menos problemas de fertilidad relacionados con involución uterina e infecciones de tracto reproductor (Butler
Cuadro 3. Efecto de grupo racial, el cambio en condición corporal y pariciones en el intervalo parto concepción (días abiertos). La Paz, El Salvador. 2004.

\begin{tabular}{lccc}
\hline \multicolumn{1}{c}{ Factor } & N & $\begin{array}{c}\text { Días } \\
\text { abiertos }\end{array}$ & $\begin{array}{c}\text { Desviación } \\
\text { estándar }\end{array}$ \\
\hline Grupo racial & & & \\
Cruzadas & 30 & 108,06 a & 33,86 \\
Holstein, & 29 & 102,0 a & 49,78 \\
Cambio en Condición & & & \\
Corporal & & & \\
0,0 - 0,49 & 15 & 97,73 a & 40,43 \\
0,5 - 0,99 & 22 & 99,31 a & 39,39 \\
$>1$ & 22 & 115,86 a & 45,56 \\
Pariciones & & & \\
Primeriza & 24 & 110,29 a & 45,10 \\
Adulta & 35 & 101,51 a & 40,33 \\
\hline
\end{tabular}

Medias con la misma letra en la misma columna no difieren significativamente, según DMS, p $<0,05$.

Cuadro 4. Efecto de grupo racial, el cambio en condición corporal y pariciones en el intervalo parto concepción (días abiertos). La Paz, El Salvador. 2004.

\begin{tabular}{lccc}
\hline Factor & N & $\begin{array}{c}\text { Dias } \\
\text { abiertos }\end{array}$ & $\begin{array}{c}\text { Desviación } \\
\text { estándar }\end{array}$ \\
\hline $\begin{array}{l}\text { Grupo racial } \\
\text { Cruzadas }\end{array}$ & 30 & $108,06 \mathrm{a}$ & 33,86 \\
$\begin{array}{l}\text { Holstein. } \\
\text { Cambio en condi- }\end{array}$ & 29 & $102,0 \mathrm{a}$ & 49,78 \\
ción corporal & & & \\
0,0 - 0,49 & 15 & $97,73 \mathrm{a}$ & 40,43 \\
0,5 - 0,99 & 22 & $99,31 \mathrm{a}$ & 39,39 \\
$>1$ & 22 & $115,86 \mathrm{a}$ & 45,56 \\
Pariciones & & & \\
Primeriza & 24 & $110,29 \mathrm{a}$ & 45,10 \\
Adulta & 35 & $101,51 \mathrm{a}$ & 40,33 \\
\hline
\end{tabular}

Medias con la misma letra en la misma columna no difieren significativamente, según DMS, $\mathrm{p}<0,05$.

y Smith 1989). En un estudio anterior se observó que las vacas que tuvieron anestros más largos, requirieron menos servicios por concepción (Corea et al. 2004). 
Aunque el efecto de la paridad en la fertilidad (Cuadro 4) parece no ser importante en las condiciones de este estudio, lo cual también ha sido descrito anteriormente en lecherías de El Salvador (Corea et al. 2004), estudios en otros países han reportado que las novillas primerizas requieren menos servicios por concepción que las vacas adultas (Cavestany 2000 b, Badinga et al. 1985) y que las vacas adultas tienen menor porcentaje de concepción que las vacas primerizas (Gwazdauskas et al. 1974, De la Sota 2001).

\section{Días abiertos}

Los efectos del grupo racial $(\mathrm{p}=0,58)$, la condición corporal $(\mathrm{p}=0,29)$ y las pariciones $(\mathrm{p}=0,39)$, no produjeron diferencias en el período entre el parto y la concepción.

Los días abiertos no tuvieron efectos significativos de los factores estudiados. La ventaja relativa de las vacas cruzadas referente a la brevedad del anestro, se pierde al tener menos fertilidad en los servicios y da como resultando que el periodo abierto sea similar al de las vacas Holstein que tuvieron mejor fertilidad. Al comparar los días abiertos entre numerosos hatos, Laben et al. (1982) tampoco encontraron diferencias entre hatos con mayores y menores producciones, ellos concluyeron que una gran parte de la razón de la mayor producción que es el manejo, contribuye a neutralizar el antagonismo entre la alta producción y desempeño reproductivo . Esto puede deberse a que un mejor manejo puede superar el efecto negativo del balance energético negativo en la fertilidad (Nebel y McGilliad 1993). Se debe tener en consideración además, que los servicios por concepción y los días abiertos son mediciones no muy claras de interpretar ya que en ellas, las decisiones de manejo se confunden con los efectos biológicos (Butler y Smith 1989).

Respecto al rol de la urea, Godden et al. (2001), monitorearon 60 hatos comerciales durante 13 meses y concluyeron que se puede alcanzar buenos resultados en la fertilidad en un amplio rango de concentraciones de urea.

Si bien el valor de los días abiertos es bastante cercano al ideal de 90 días y mejora la meta práctica de 120 días en las dos fincas estudiadas; es necesario estudiar el papel de otras determinantes de la reproducción no incluidas en este estudio; por ejemplo producción individual, enfermedades como mastitis, metritis, cojeras, el rol del balance nutricional en la dieta ofrecida y el ambiente.

Debido a las condiciones propias del estudio, al limitado número de animales y la variabilidad entre ellos, no se pudieron establecer vínculos conocidos como la relación entre condición corporal y las pariciones con la fertilidad.

\section{CONCLUSIONES}

La genética Holstein y la mayor pérdida de condición corporal tienen un efecto negativo en el reinicio de la actividad ovárica de las vacas.

A pesar de que los niveles de nitrógeno ureico en sangre son buenos indicadores de la nutrición proteica, no tienen una relación clara con el desempeño reproductivo ya que en este estran en juego otros factores como la energía en la dieta y el manejo reproductivo.

Los días abiertos son influenciados por la duración del anestro y por la habilidad del inseminador. Existe aún una importante oportunidad para mejorar este periodo en ganaderías similares a las de este estudio si se mejora la efectividad de la técnica de inseminación artificial.

\section{AGRADECIMIENTOS}

Este estudio fue realizado con el apoyo financiero de la Agencia Internacional de Energía Atómica (proyecto ELS 5009), La Fundación Internacional para la Ciencia (proyecto AB- 14133) y el Consejo de Investigaciones Científicas de la Universidad de El Salvador (proyecto 02-54).

Se agradece a los Doctores Ana Meikle y Daniel Cavestany de la Universidad de la República en Uruguay por sus aportes a este trabajo y a los propietarios de las lecherías por su colaboración. 


\section{LITERATURA CITADA}

Bach, A. 2002. La reproducción del vacuno lechero: Nutrición y fisiología. Producción Animal N 175. Pag 13-41.

Badinga, L; Collier, RJ; Thatcher, WW; Wilcox, CJ. 1985. Effects of climatic and management factors on conception rate of dairy cattle in subtropical environment. J Dairy Sci 68: $78-85$.

Baker, LD; Ferguson, JD, Chalupa, W. 1995. Responses in urea and true protein of milk to different protein feeding schemes for dairy cows. University of Pennsylvania, USA. P. $2425-2433$.

Bolaños, JM; Molina, JR. 1992. Efecto del amamantamiento restringido sobre la actividad ovárica de vacas cebú. Ciencias Veterinarias (Costa Rica) 14: 30-35.

Butler, WR; Smith, RD. 1989. Interrelationships between energy balance and postpartum reproductive function in dairy cattle. J Dairy Sci 72: 767-783.

Butler, WR. 1998. Effect of protein nutrition on ovarian and uterine physiology in dairy cattle.J Dairy Sci. 8:25332539 .

Carrol, DJ; Barton, BA; Anderson, GW; Smith, RD. 1988. Influence of protein intake and feeding strategy on reproductive performance of dairy cows. J Dairy Sci. 71:3470-3481.

Cavestany, D. 2000a. Reinicio de la actividad ovárica posparto mediante la determinación de los niveles de progesterona en leche. En Serie Técnica 116. INIA, La Estanzuela. Uruguay. p. 25.

Cavestany, D. 2000b. Diferentes esquemas de sincronización de celos. In: Serie Técnica 116. INIA, La Estanzuela. Uruguay. p. 33.

Coleman, DA; Thayne, WV; Dailey, RA. 1985. Factors affecting reproductive performance of dairy cows. J Dairy Sci 68: 1793 - 1803.

Corea, EE; Silva, O; Alvarado, JF; Leyton, LV; Castillo, GO; López, LH; Sandoval, A; Platero, CR; Erroa, RM. 2004. Evaluación del anestro posparto y estudio de los parámetros reproductivos en ganado lechero en El Salvador. Informe Técnico. Universidad de El Salvador-Organismo Internacional de Energía Atómica. 100 p.

De la Sota, RL.; BO, G; Gallardo, M. 2001. Reproducción en rodeos de leche. Curso de posgrado en reproducción bovina. Instituto de Reproducción Animal Córdoba. Argentina. p. 42-49

Delgado R; Magaña JG; Galina, C; Segura, JC. 2004. Effect of body condition score at calving and its changes during early lactation on postpartum reproductive performance of zebu cows in a tropical environment. J. Appl. Anim. Res. 26:23-28.

Domecq, JJ; Skidmore AL; Lloyd, JW; Kaeene, JB. 1997a. Relationship between body condition scores and milk yield in a large dairy herd of yielding Holstein cows. J Dairy Sci 80: 101 - 112 .

Domecq, JJ; Skidmore AL; Lloyd, JW; Kaeene, JB. 1997b. Relationship between body condition scores and conception at first artificial insemination in a large dairy herd of high yielding Holstein cows. J Dairy Sci 80: $113-120$.

Edmonson, AJ; Lean, IJ; Weaver, LD; Farver, T; Webster, G. 1989. A body condition scoring chart for Holstein dairy cows. J Dairy Sci 72: 68 - 78 .

Ferguson JD; Chalupa W. 1989. Impact of protein on reproduction in dairy cows. J Dairy Sci. 72:746-766.

Ferguson JD; Galligan TB; Blanchard T; Reeves M. 1993. Serum urea nitrogen and conception rate: The usefulness of test information. J Dairy Sci. 76:37423746 .

Flamenbaum, I. 1997. Management of dairy cows in hot climate conditions. In: curso internacional de ganadería lechera intensiva en diferentes condiciones de producción. Shefayim, Israel. p. 1.

Godden, SM; Kelton, DF; Lissemore, KD; Walton, JS; Leslie, KE; Lumsden JH. 2001. Milk Urea testing as a tool to monitor reproductive performance in Ontario dairy herds. J. Dairy Sci. 84: 1397-1406. 
Gwazdauskas, FC; Wilcox, CJ; Thatcher, WW. 1974. Environmental and managemental factors affecting conception rate in a subtropical climate. J Dairy Sci 58: $88-91$.

Hof, G; Vervoorn, MD; Lenaers, PJ; Tamminga, S. 1997. Milk urea nitrogen as tool to monitor the protein nutrition of dairy cows. J Dairy Sci 80: 33333 - 3340 .

Hojman, D; Krog, O; Adin, G; Gips, M; Hanochi, B; Ezra, E. 2004. Relationships among milk urea production, nutrition and fertility taits in Israeli dairy herds. J. Dairy Sci. 87: 1001-1011.

Jordan, ER; Chapman, TE; Holtan, DW; Swanson, LV. 1983. Relationship of dietary crude protein to compositions of uterine secretions and blood in high producing post partum dairy cows. J Dairy Sci 66: 1854 - 1862.

Kim, IH; Suh, GH. 2003. Effect of the amount of body condition loss from the dry to near calving periods on the subsequent body condition change, occurrence of postpartum diseases, metabolic parameters and reproductive performance in Holstein dairy cows. Theriogenology 60(8): 1445-56.

Latrille, L. 1993. Nutrición y reproducción en la vaca lechera. Avances en Producción Animal 18: 3-20.

Laben, RL; Shanks, R; Berger, PJ; Freeman, AE. 1982. Factors affecting milk yield and reproductive performance. J Dairy Sci 65: 1004 - 1015.

Lucy, MC; Staples, CR; Thatcher, WW; Erickson, PS; Cleale, RM; Firkins, JL; Clark, JH; Murphy, MR; Brodie, BO. 1992. Influence of diet compositions, dry matter intake, milk production and energy balance on time of postpartum ovulation and fertility in dairy cows. Anim. Prod. 54: 323 - 331.

Melendez, P; Donovan, A; Hernandez, J. 2000. Milk urea nitrogen and fertility in Florida Holstein cows. J Dairy Sci. 83: 459-463.
Nebel, RL; Mcgilliard, ML. 1993. Interactions of high milk yield and reproductive performance in dairy cows. J Dairy Sci 76: 3257 - 3268.

Pryce, JE; Coffey, MP; Simm, G. 2001. The relationship between body condition score and reproduction performance. J. Dairy Sci. 84: 1508-1515.

Randel, RD. 1990. Nutrition and postpartum rebreeding in cattle. J Dairy Sci 68:853-862.

Raheja, KL; Burnside, EB; Schaeffer, LR. 1989. Relationship between fertility and production in Holstein dairy cattle in different lactations. J Dairy Sci 72: 2670 - 2678.

Ropstad, E; Refsdal, AO. 1987. Herd reproductive performance related to urea concentration in bulk milk. Acta vet. Sand. 28: $55-63$.

Ruegg, PL; Goodger, WJ; Holmberg, CA; Weaver, LP. Huffman, EM. 1992. Relation among body condition score, serum urea nitrogen and cholesterol concentrations and reproductive performance in high producing Holstein dairy cows in early lactation. Am J Vet Res 53: 10 - 13.

Staples, CR; Thatcher, WW; Clark, JH. 1990. Relationship between ovarian activity and energy status during the early postpartum period of high producing dairy cows. J. Dairy Sci. 73:938-947.

Whitmore, HL; Tyler, WJ; Casida, LD. 1974. Efects of early postpartum breeding in dairy cattle. J Anim Sci. 38: 339.

Zarco, L.A. 2000. Efectos del balance energético en la reproducción de la vaca lechera de alta producción: mecanismos, importancia y prevención. In: Mejoramiento animal y reproducción. 2 ed. UNAM, México. p. 151.

Zavala DE; López, FM; Ventura, B; Corea, EE. 2005. Efecto de la energía y la proteína en la fertilidad de vacas lecheras en ocho lecherías de El Salvador. Tesis. Ing Agr. Universidad de El Salvador. 120 p. 